\title{
EFFICACY OF PERMEATAL ENDOSCOPIC MYRINGOPLASTY IN INACTIVE (MUCOSAL) CHRONIC OTITIS MEDIA
}

\author{
Saleem Asif Niazi, Shahzad Maqbool, Yousra Riaz, Zafarullah Khan, Shafaq Zaman, Rubab Akhtar \\ Pak Emirates Military Hospital/National University of Medical Sciences (NUMS) Rawalpindi Pakistan
}

\section{ABSTRACT}

\begin{abstract}
Objective: To determine the efficacy of permeatal ednoscopic myringoplasty, in inactive (mucosal) COM regarding, graft take and hearing improvement.

Study Design: Quasi-experimental study.

Place and Duration of Study: ENT Department, Pak Emirates Military Hospital, Rawalpindi, from Aug 2019 to Feb 2020.

Methodology: This was conducted on a sample size of $\mathrm{n}=100$. Patients having inactive (mucosal) COM with dry central small, medium and large perforations were included. Patients were divided in to three groups on the basis of age, group A 15-30 years, group B 31-45 years and group C 46-60 years. All the patients underwent per-meatal endoscopic myringoplasty, without raising the tympano-meatal flap, under general anesthesia. Patients were followed up over a period of six months. Graft take was assessed by microscopy. Hearing was assessed by Pure Tone Audiometry (PTA) with improvement in air bone gap to $15 \mathrm{db}$ as primary end point. Data was collected on structured performa. Data was analyzed using SPSS version 17.

Results: In this study, 33 (33\%) were males and $67(67 \%)$ were females. Age ranged 15-60 years. Out of 5 patients lost to follow up. A total of 95 completed their follow up. Based on age of the patients, in group A, successful graft take was 100\%. In group B, it was $91 \%$ \& in group C it dropped to $46 \%$. On the basis of tympanic membrane perforation size, the graft success rate in small perforations was $94.8 \%$; in medium sized perforations it was $86.8 \%$ and in large sized perforations of tympanic membrane it was $61.1 \%$.

Conclusion: Permeatal endoscopic myringoplasty is a reliable, relatively swift procedure with lesser post operative morbidity. It has overall success rate of $81.3 \%$. This technique should be encouraged for further research, to contemplate results on larger sample size.
\end{abstract}

Keywords: EES, Endoscopic ear surgery, Pop in technique, Permeatal myringoplasty.

How to Cite This Article: Niazi SA, Maqbool S, Riaz Y, Khan Z, Zaman S, Akhtar R. Efficacy of Permeatal Endoscopic Myringoplasty in Inactive (MUCOSAL) Chronic Otitis Media. Pak Armed Forces Med J 2021; 71 (Suppl-3): S552-555. $\quad$ Doi: https://doi.org/10.51253/pafmj.v1i1.7915

This is an Open Access article distributed under the terms of the Creative Commons Attribution License (https://creativecommons.org/licenses/by-nc/4.0/), which permits unrestricted use, distribution, and reproduction in any medium, provided the original work is properly cited.

\section{INTRODUCTION}

Chronic Otitis Media (COM) previously called Chronic Supurative Otitis Media (CSOM) is one of the most common and frequent ear disorder encountered in ENT OPD, especially in developing countries like Pakistan. ${ }^{1-3}$ It can be managed conservatively as well as surgically. A persistent dry ear, for 4-6 weeks can be managed surgically via grafting mainly temporalis fascia, by the surgical technique named myringoplasty. Myringoplasty not only converts the diseased ear into a safe ear, but also improves hearing and quality of life. ${ }^{4}$

Before the advent of Endoscopic Ear Surgery (EES) in 1990s, myringoplasty was a microsurgical procedure, and could be performed by, i.e., per-meatal, end-aural, or post-aural approach. Tympano-meatal flap was raised in all the above approaches. Now, in the era of EES, per-meatal/trans-canal approach is used, tympano-meatal flap is mostly raised \& perforation is grafted. Its efficacy can be measured in terms of

Correspondence: Dr Saleem Asif Niazi, HOD ENT Department, Pak Emirates Military Hospital, Rawalpindi Pakistan lesser morbidity, shorter operating time, reduced post operative hospital stay, and the surgical scar for graft harvesting, is small and hides well under the hairline.

We conduct this study to assess the results of gra$\mathrm{ft}$ success with regards to size of perforation and age of patients; in this per-meatal endoscopic technique of myringoplasty, in which the tympano-meatal flap is not raised \& temporalis fascia graft is popped-in.

\section{METHODOLOGY}

A quasi-experimental study was conducted from August 2019 to February 2020, in ENT Department of Pak Emirates Military Hospital, Rawalpindi. Sample size was $n=100$; inducted by convenience sampling. Approval was taken from the hospital's Institutional Review Board. Written consent was obtained from all patients inducted. Data was collected on a structured performa, including demographic details and outcomes of surgery.

Inclusion Criteria: Patients between the ages of 15 and 60 , with central small $(<25 \%$ of the tympanic membrane), medium (more than $25 \%$ of tympanic 
membrane) and large perforations (up to $50 \%$ of the tympanic membrane); discharge free for more than 4-6 weeks were included.

Exclusion Criteria: Comprise of patients having total or subtotal tympanic membrane perforations, active mucosal COM, squamous epithelial type of COM, profound sensory-neural hearing loss, gross DNS, any systemic illness like diabetes or hypertension, and extreme of ages.

As per protocol, all patients were admitted to inpatient facility one day prior to surgery, preceded by relevant work up. Thorough ENT examination and microscopic examination (EUM) of the ear and eustachian tube assessment by endoscopy, under local anesthesia was carried out. Pure tone audiometry (PTA) and x-rays of mastoids were also requested. Patients were placed in three groups with respect to age, group A 15-30 years, group B 31-45 years and group C 46-60 years.

Endoscopic per-meatal myringoplasty of all included candidates was done under general anesthesia. Temporalis fascia graft was harvested from 3-4 cm hairline surgical incision. Zero degree rigid endoscopes of $2.7 \mathrm{~mm}$ and $4 \mathrm{~mm}$ in diameter, and $16-18 \mathrm{~cm}$ in length were introduced in the external auditory canal (EAC). Margins of perforation were freshened using postage stamp technique. Middle ear cavity including eustachian tube opening was assessed endoscopically. Gelfoam was filled in the middle ear cavity and graft of appropriate size was tucked in all around the tympanic membrane remnant. We named it as 'Pop-in Technique'. EAC was packed with dry gelfoam, and mastoid dressing applied. Postoperatively intravenous antibiotics cover for minimum of 3 days was given and then oral antibiotics prescribed for another 4 days. Follow up was planned at the end of first, $6^{\text {th }}$ and $12^{\text {th }}$ weeks post operatively. On each visit, EUM of operated ear was carried out to access the graft take. Pot operative hearing was assessed by PTA with improvement in air bone gap up to $15 \mathrm{db}$ as primary end point. Persistence of perforation and no improvement in hearing were taken as failed outcome. Data was analyzed using SPSS-17 and the outcome of endoscopic per-meatal myringoplasty was the dependant variable. Quantitative variables expressed as mean with $\pm \mathrm{SD}$, whereas qualitative data was expressed in percentage and frequencies. $p$-value was generated using chi-square.

\section{RESULTS}

Study was conducted on 100 patients, with the female preponderance of $n=67(67 \%)$ whereas only
$33 \%$ were males. Age range was from $15-60$ years, with mean of $35.95 \pm 12.4$. Out of 5 patients lost to follow up (one from group A, two each from groups B \& C). A total of 95 completed their three months follow up. In group A, 34 patients (100\%) had a successful graft take. In group B, 40 cases $(91 \%)$ out of $n=44$ had a successful graft take, while only $4(9 \%)$ cases were failure. In group C, 7 out of 15 (46\%) cases had a successful graft take and remaining $n=8(53 \%)$ had graft failure. (Figure-1).

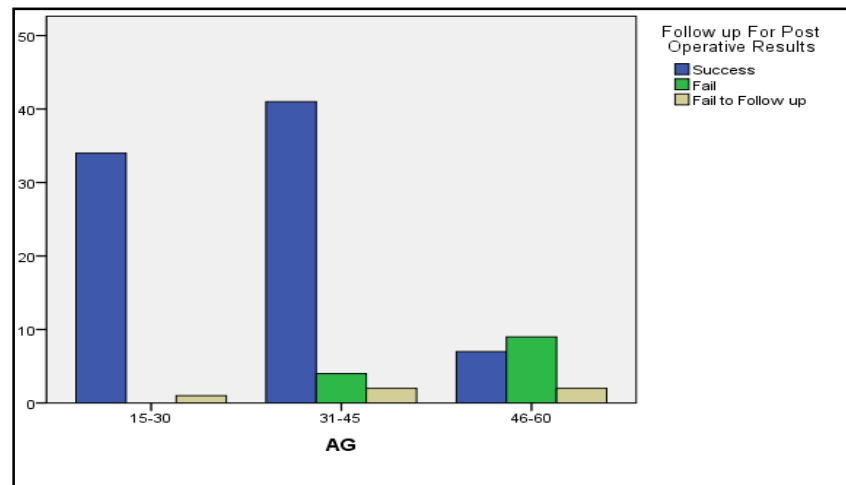

Figure-1: Significant inverse relation of age group and graft take.

On the basis of the size of tympanic membrane perforation, small and medium sized perforations were present in 40 patients each. While $n=20$ patients had large perforations. Graft success rate in small perforations was $37 / 39$ cases $(94.8 \%)$; in medium sized perforations it was $34 / 38$ cases $(89.4 \%)$ and in large sized perforations of tympanic membrane it was $11 / 18$ cases $(61.1 \%)$ (Table). Age of the patient and size of perforation had an inverse relation with the graft take success; with statistically significant $p$-values of $<0.05$ each. The overall success rate of graft take in our study was $81.3 \%$.

Table: Comparison of size of perforation and graft take.

\begin{tabular}{l|c|c|c|c}
\hline \multicolumn{7}{|c|}{ Follow up For Post Operative Results } & \multirow{2}{*}{ Total } \\
\cline { 1 - 4 } & Success & Fail & Fail to Follow Up & \\
\cline { 1 - 4 } Size of Perforation & \multicolumn{3}{c}{} \\
\hline Small & 37 & 2 & 1 & 40 \\
\hline Medium & 34 & 4 & 2 & 40 \\
\hline Large & 11 & 7 & 2 & 20 \\
\hline Total & 82 & 13 & 5 & 100 \\
\hline
\end{tabular}

\section{DISCUSSION}

Inactive (mucosal) $\mathrm{COM}$ has been classified by WHO as Chronic Suppurative Otitis Media (CSOM). In this type of COM, pars tensa has a permanent perforation but the mucosa of middle ear cleft is not inflamed. The patients usually present with hearing loss and a central dry perforation. 
Tympanoplasty refers to the eradication of the disease and grafting of the tympanic membrane and/ or reconstruction of the ossicular chain if necessary. Myringoplasty entails tympanoplasty without ossicular reconstruction. Myringoplasty was first mentioned in history in 1878, but was properly documented by German surgeons latter in $1952^{5}$. Horst Wullstein wrote proper classification of middle ear repair in $1958 .{ }^{6}$ He also introduced operating microscope, a transformative event in the ear surgery, ${ }^{7}$ Objectives of myringoplasty are essentially to restore the integrity of tympanic membrane and to improve hearing, ${ }^{8}$ Myringoplasty is an established procedure and performed by various approaches and techniques. Underlay technique using temporalis fascia or sometimes perichondrium, by end/post-aural approach is most commonly practiced. However, the quest is to improve the results, operating time and postoperative recovery period, by analyzing novel otological surgical techniques.

Endoscopic myringoplasty has been introduced for in this endeavor, since 1990s. ${ }^{9}$ There was one earlier report in 1992 of endoscopic myringoplasty from El-Guindy. ${ }^{10}$ Endoscopes allow for enhanced surgical visualization; and have transformed EAC into an operative gateway. The advantages of Endoscopic Ear Surgery (EES) include, wider view, enhanced imaging \& magnification, minimally invasive technique with better ability to perform reconstructive procedures, ${ }^{7}$ shorter operating time as compared to microscope assisted surgery, lesser complications and rapid post operative recovery translating into lesser hospital stay. EES has become an emerging avenue that has gathered a significant amount of interest. The endoscopic surgery can be applied in a spectrum of otological procedures such as grommet insertion, all five types of tympanoplasty, ossicular reconstruction, cholesteatoma clearance, stapedectomy and cochlear implant surgery.

Graft placement can either be medial or lateral to tympanic membrane remanents. ${ }^{11}$ Medial grafting, also known as the underlay technique, is most commonly practiced. This technique involves raising a tympanomeatal flap through a canal incision and raising the annulus and tympanic membrane remnants. Graft is then slipped between the bony EAC \& the raised tympanomeatal flap. Gel foam is already tucked in the middle ear cavity, ${ }^{12}$ to keep the graft lateralized. The graft can be positioned either medial or lateral to the handle of maleus making no difference. Materials used for grafting can be temporalis muscle fascia, tragal perichondrium, tragal or conchal cartilage and even allografts. ${ }^{13}$

In our study, we resorted to EES, for inactive (mucosal) COM, a technique not commonly mentioned in literature. Rigid endoscopes, $2.7 \mathrm{~mm}, 0^{\circ}$ and 16-18 $\mathrm{cm}$ in length were used. The technique is unique in a way that the tympano-meatal flap is not raised and tucking in the temporal fascia graft all around the deepithelized margins of the tympanic membrane perforation (Figure-2). EES is mostly advocated in cases

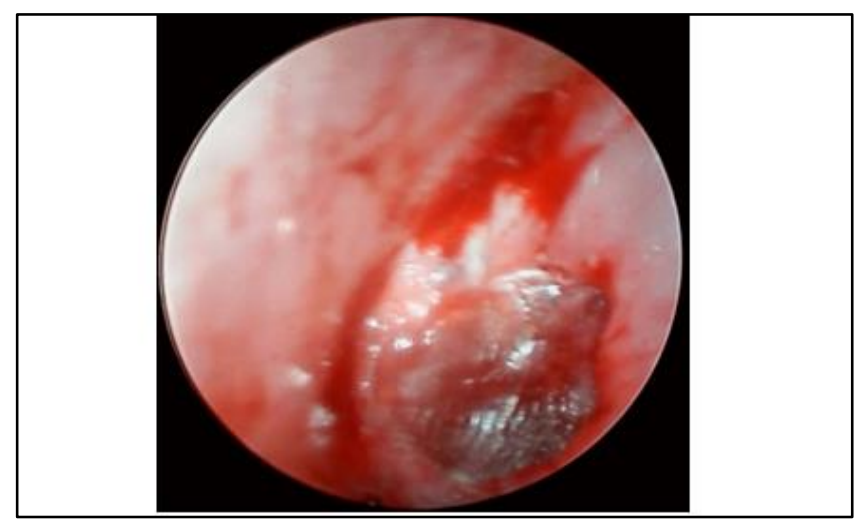

Figure-2: Tucking in of temporalis fascia graft all around the margins of perforation.

of small size tympanic perforations with wide EAC. However, in our study small, medium and large perforations irrespective of EAC circumference were grafted. Permeatal/trans-canal endoscopic myringoplasty, without raising the tympano-meatal flap (Pop-in Technique) though difficult, due to lesser space of maneuverability in EAC; has proved to be an efficacious technique with lesser morbidity, shorter operative time \& post operative hospital stay, cosmetically superior with a small scar in the hairline. Occam's razor encourages the procedure which is simpler, swift \& carries lesser morbidity. ${ }^{14}$

We were able to establish an inverse relation of graft take with age of patients \& size of perforation. Ears with small perforation had $94.8 \%$ graft take. Next was medium sized perforation with a graft take of $86.8 \%$. While large perforations showed only $61.1 \%$ success in graft takes. Our results are comparable to the study conducted in Egypt, by Ahmed El- Guindy in 1992, who gave graft success rate of $91 \%$.

With an overall success rate of graft take $81.3 \%$, our Pop-in Technique stands comparable to the conventional microscope assisted myringoplasty using underlay technique. In 2001, Raj and Mehar reported a success rate of $90 \%$. Likewise, later in 2015, Patel et al, 
reported a comparable success rate in patients undergoing endoscopic transcanal tympanoplasty. ${ }^{15}$

Our study had a few limitations. Sample size $(n=100)$ was not large enough to yield more reliable results, yet we were able to establish generalized results for our target population. A comparative study with microscopic tympanoplasty would have more varied results. The Pop-in technique though very less discussed in literature, was adopted by the senior author, in 2006, and managed to publish an article of his experience in 2013. ${ }^{16}$ So forth, we believe that this study will encourage the otologists of endoscopic era to work and adopt better approaches for ear surgery and thus generate even better results. Otoendoscopy is the future of myringoplasty and tympanoplasty. ${ }^{17}$ Endo-scopic ear surgery has super added benefit of being less invasive as compare to microscopic myringoplasty. ${ }^{18}$

\section{CONCLUSION}

Permeatal endoscopic myringoplasty (Pop in technique) is a reliable, relatively swift procedure with lesser post operative morbidity especially in small and medium sized perforation. It has overall success rate of $81.3 \%$. This technique should be encouraged for further research, to contemplate results on larger sample size.

\section{Conflict of Interest: None.}

\section{Authors' Contribution}

SAN: Concept, study design, revision, SM: Data interpretation, write up, YR: Literature search, data acquisition, ZK: Data collection \& analysis, SZ: Data acquisition \& analysis, RA: Data acquisition \& analysis.

\section{REFERENCES}

1. Acuin J. Chronic suppurative otitis media: Burdon of illness and management options in Child and adolescent health and development prevention of blindness and deafness: World Health Organisation, Geneva, Switzerland. 2004, 1-84. Available at: https://apps.who.int /iris/handle/10665/42941

2. Hussain A, Yousaf N, Khan AR. Outcome of Myringoplasty. J Postgrad Med Inst Dec 2004; 18(4): 693-696.

3. Sunderman J, Dyer H. Chronic ear disease in Australian Aborigines. Med J Aus 1984; 140(1): 708-711.
4. Rance W, Raney MD. Myringoplasty and tympanoplasty BCM (Baylor College of Medicine) Bobby R Alford. Department of Otolaryngology-Head and Neck Surgery. 1995.

5. Mudry A. History of myringoplasty and tympanoplasty type I Otolaryngol Pol 2014; 68(1): 30-33.

6. Wullestein H. Theory and practice of tympanoplasty. Laryn 1956; 66(1): 1076-1095.

7. Kapadiya M, Tarabichi M. An overview of endoscopic ear surgery in 2018. Laryngoscope Investig Otolaryngol 2019; 4(3): 365373.

8. Ashfaq M, Aasim MU, Khan N. Myringoplasty: Anatomical and functional results. Pak Armed Forces Med J 2004; 54(2): 155-158.

9. Patel J, Aiyer RJ, Gajjar Y, Gupta R, Raval J, Suthar PP. Endoscopic tympanoplasty vs microscopic tympanoplasty in tubotympanic CSOM: a comparative study of 44 cases. Inter J Res Med Sci 2015; 3(8): 1953-1957.

10. El-Guindy A. Endoscopic transcanal myringoplasty. J Laryngol Otol 1992; 106(6): 493-495.

11. Manual of middle ear surgery vol-1 by Mirko Tos. Published by thieme medical publishers Inc, Newwork. 1993: 184. [Internet] Available at: https://www.amazon.com/Manual-Middle-EarSurgery-1-ebook/dp/B01CDOZV9K

12. Janiak-Kiszka J, Kaźmierczak W. The results of myringoplasty using underlay technique. Otolaryngol Pol 2014; 68(1): 30-33.

13. Javaid M, Iqbal M, Shah HJI. Myringoplasty, onlay versus underlay technique. J Postgrad Med Inst 2002; 16(2): 174-177.

14. Halim A, Borgstein J. Pediatric myringoplasty: postaural versus transmeatal approach. Inter J Paediatric Otolaryngol 2009; 73(2); 1580-1583

15. Patel J, Aiyer RG, Gajjar Y, Gupta R, Raval J, Suthar PP. Endoscopic tympanoplasty vs microscopic tympanoplasty in tubotympanic CSOM: a comparative study of 44 cases. Int J Res Med Sci 2015; 3(8): e1953-e1957.

16. Niazi SA, Hassan ZU, Atif K, Ullah S. Comparision of Permeatal medial placement of graft without raising the tympanomeatal flaps to conventional methods of myringoplasty: an experience at tertiary care hospital in Pakistan. Pak J Med Sci 2016; 32(4): $927-$ 930.

17. Jyothi AC, Shrikrishna AC, Kulkarni NH, Kumar A. Endoscopic myringoplasty versus microscopic myringoplasty in tubotympanic CSOM: a comparative study of 120 cases. Indian J Otolaryngol Head Neck Surg2017; 69(3): 357-362.

18. Tseng CC, Lai MT, Wu CC, Yuan SP, Ding YF. Endoscopic transcanal myringoplasty for tympanic perforations: An outpatient minimally invasive procedure. Auris Nasus Larynx 2017; 45(3): 433-439. 